\title{
CASE REPORT: TUBERCULOUS SACROILIITIS
}

\author{
Maria Lara Picolo1, ${ }^{1,}$, Poliane Cruz Costa ${ }^{1}$, Ângela Massignan ${ }^{1}$, Marcelo Wainberg Jeffman $^{1}$, Carolaine de Oliveira ${ }^{2}$ \\ 1.Hospital Moinhos de Vento, Porto Alegre (RS), Brazil; 2 Universidade Luterana do Brasil, Canoas (RS), Brazil. \\ *Corresponding author: mlarapicolo@gmail.com
}

\section{BACKGROUND}

Bone and joint tuberculosis reaches up to $35 \%$ of cases of extrapulmonary tuberculosis (TB), especially in immunosuppressed patients. Skeletal TB most often involves the thoracic spine (Pott's disease), followed by tuberculous arthritis in weight-bearing joints and extraspinal TB osteomyelitis. The sacroiliac joint (SI) is an uncommon site of infection and its diagnosis is often delayed.

\section{CASE REPORT}

A 37-year-old woman, housewife, with no comorbidities, presents with a 6-month progressive inflammatory low back and right gluteal pain. During this time, she used several anti-inflammatory drugs with partial improvement of symptoms and frequent recurrence. Two weeks before admission, she started presenting evening fever and night sweats and was treated with amoxicillinclavulanate for seven days, with no improvement in symptoms. There was a loss of $5 \mathrm{~kg}$ throughout the period. She came to the emergency with disabling low back pain, no respiratory, genitourinary or gastrointestinal symptoms. Her physical examination revealed stable vital signs, pain in SI topography, and positive Patrick's and Gaenslen's tests. Laboratory tests were performed showing mild normocytic normochromic anemia, high levels of erythrocyte sedimentation rate and C-reactive protein, negative viral serology. Magnetic resonance imaging (MRI) of sacroiliac joints revealed sacroiliitis with inflammatory and infectious features (Figure 1). A computed tomography (CT) was carried out to guide a biopsy, also showing bone erosions (Figure 2). A chest CT demonstrated a miliary pattern (Figure 3). Joint fluid showed a rapid test (PCR) for Mycobacterium tuberculosis and negative acid-fast bacillus (AFB) test. Mycobacterium tuberculosis culture and IGRA (interferon gamma release assay) were performed with a positive result. Treatment with rifampicin, isoniazid, pyrazinamide and ethambutol were started.

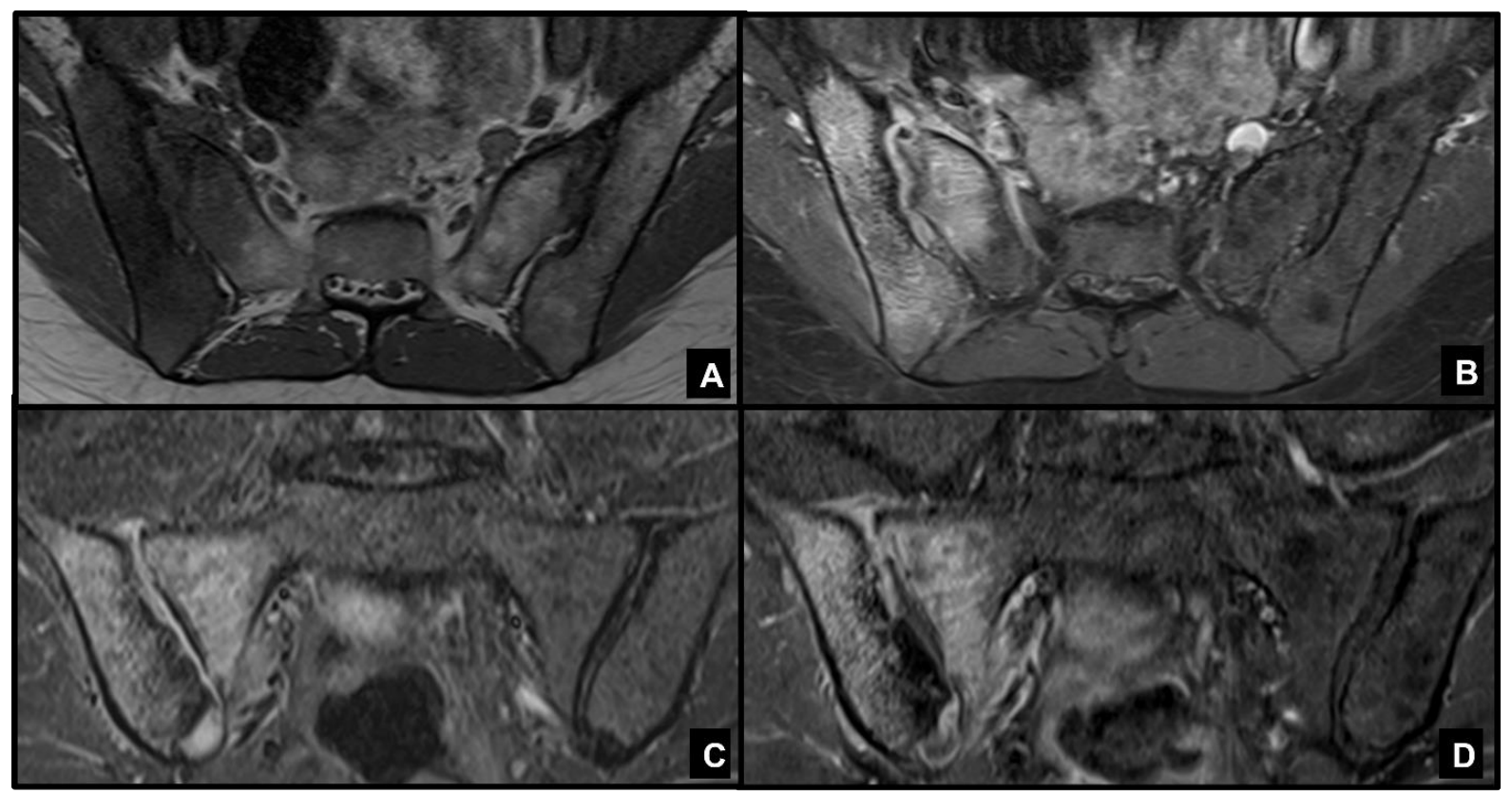

Figure 1. Magnetic resonance imaging of the SI. Oblique axial T1-weighted (A) and fat-suppressed contrast enhanced T1-weighted (B); oblique coronal STIR (C) and fat-suppressed contrast-enhanced T1-weighted (D) images of SI show small amount of right sacroiliac joint effusion associated with anterior and superior capsular bulging, synovitis, bone erosions, bone marrow edema and enhancement in pericapsular soft tissue and along right gluteus.

Realização: 


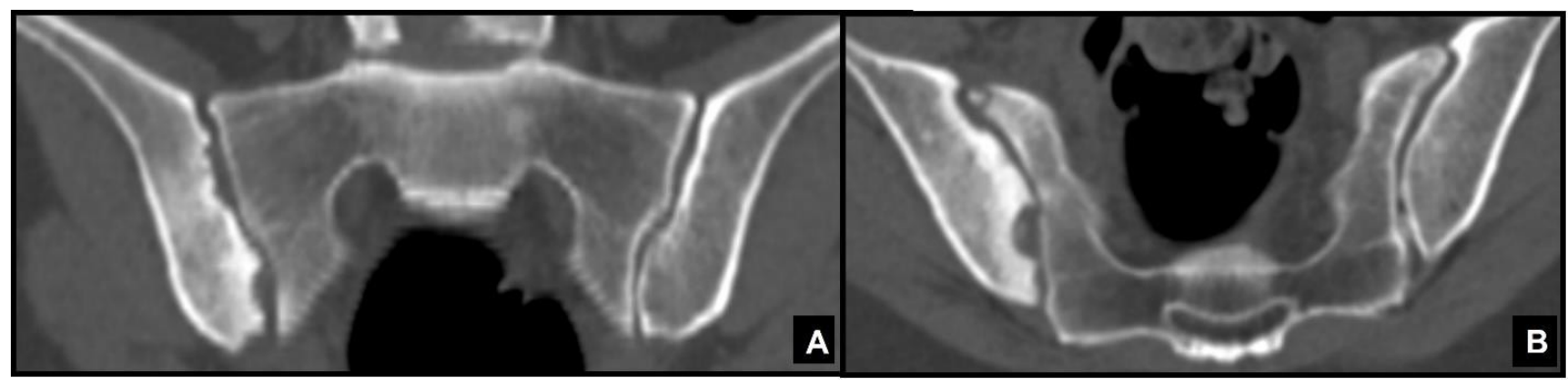

Figure 2. Sacroiliac CT. Oblique coronal (A) axial (B) show large bone erosions and subchondral sclerosis in right SI.

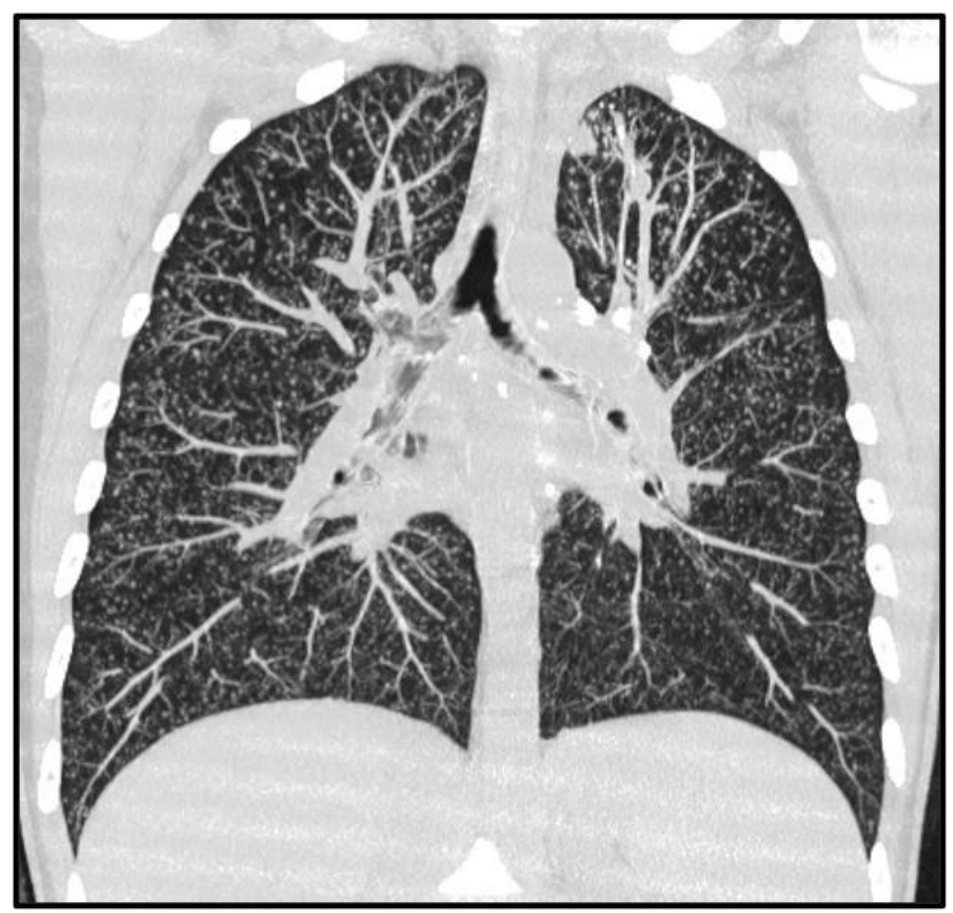

Figure 3. Chest CT. Coronal maximum-intensity-projection image showing miliary pulmonary nodules.

\section{CONCLUSION}

Sacroiliitis is a main sign of axial spondyloarthropathies; however, some clinical signs and symptoms associated with suggestive imaging findings may indicate an infectious etiology. A correct diagnosis is essential, mainly because delayed or misleading diagnosis and treatment can lead to joint destruction. In addition, the unnecessary use of immunosuppressants can result in serious complications.

\section{KEYWORDS}

Tuberculosis, Sacroiliitis, Infectious sacroiliitis, Imaging sacroiliac. 